\title{
Screening of Influenza A (H1N1) neuraminidase inhibitor for Kabasura Kudineer, Nilavembu Kudineer and the novel formulation JACOM
}

\author{
Shree Devi M.S ${ }^{1}$, Sathiyarajeswaran. $P^{1}$, Mohanasrinivasan $V^{1}$, Subathra Devi $C^{2}$, \\ Ramaswamy RS $^{3}$, Kanakavalli $K^{4}$
}

1. Siddha Central Research Institute, (CCRS), Arumbakkam, Chennai, Tamil Nadu, India

2. School of Bioscience and Technology, VIT University,

Vellore, Tamil Nadu, India

3. Former Director General, Central Council for Research in Siddha Chennai, Tamil Nadu, India.

4. Director General, Central Council for Research in Siddha Chennai, Tamil Nadu, India.

\begin{abstract}
Due to erratic climate change, vector-borne diseases started flaring up from the second half of the last decade. Siddha medicine has been used as a public health tool to effectively manage chikungunya and dengue in the epidemics that happened in 2008 and 2016. Tamil Nadu government has made enormous efforts to control vector-borne diseases. Due to which morbidity and mortality due to vector borne diseases came down compared with other states. Two official Siddha formulations, namely Kabasura Kudineer Chooranam and Nilavembu Kudineer Chooranam and novel herbal formulation - JACOM, are used to combat vector-borne diseases. These decoctions lack an evidence base as a formulation. Screening has been done to check the efficacy of the formulation in inhibiting neuraminidase. Neuraminidase inhibition assay was performed to determine the activity of Siddha formulations. The Kabasura Kudineer Chooranam, Nilavembu Kudineer Chooranam and JACOM showed excellent inhibitory activity. The Kabasura Kudineer and Nilavembu Kudineer and JACOM aqueous extract showed maximum neuraminidase inhibition of 80.35\%, 91.78\% and $87.97 \%$, respectively.
\end{abstract}

Keywords: Neuraminidase inhibition assay, Kabasura Kudineer Chooranam, Nilavembu Kudineer Chooranam, JACOM, H1N1, Influenza A 


\section{Corresponding Author:}

Dr. M. S. Shree Devi M.D(Siddha), Ph.D

Research Officer (S),

HoD, Dept of Pharmacy,

Siddha Central Research Institute (CCRS),

Ministry of AYUSH, Arumbakkam, Chennai- 106.

shreemd@gmail.com / ms.shreedevi@gov.in

+919443056180.

\section{Introduction}

The effect of climate change on the distribution and intensity of vector-borne diseases has been a controversial topic for the last 20 years. Climate change might increase risk areas for infectious diseases like dengue fever and increase the burden of common diseases, putting many individuals in danger [1]. Climate change has impacted sudden disaster in Chennai during 2015, causing heavy floods raising the incidence of vector-borne diseases. However, proper action of the government and dispensing of Nilavembu Kudineer as prevention has inhibited the virulence of vector-borne diseases [2]. Nilavembu Kudineer has an antiviral property which is used by people long back. Kabasura Kudineer is also used to treat vectorborne diseases, but it has been used to treat Swine flu. However, there is no existing evidence to support the claim of using these decoctions in swine flu. Andrographolide from Andrographis paniculata has been tested for its efficacy of inhibiting neuraminidase earlier [3]. However, Nilavembu Kudineer, Kabasura Kudineer and JACOM as a formulation have not been tested. The present research will warrant justice to the anti-influenza therapy from Siddha products. The study's main aim is to evaluate the neuraminidase inhibition activity of Kabasura Kudineer Chooranam, Nilavembu Kudineer Chooranam and JACOM.

\section{MATERIALS AND METHODS}

Kabasura Kudineer Chooranam is a polyherbal Siddha formulation consisting of fifteen ingredients (Tale.1), Nilavembu Kudineer Chooranam consists of nine ingredients (Table.2), and JACOM formulation consists of five ingredients (Table.3). Kabasura Kudineer Chooranam, Nilavembu Kudineer Chooranam and JACOM procured from GMP Siddha Central Research Institute Pharmacy, Arumbakkam, Chennai - 600106. Tamil Nadu, India. 
TABLE: 1 Kabasura Kudineer Chooranam.

\begin{tabular}{|c|c|c|c|}
\hline S.NO & TAMIL NAME & SCIENTIFIC NAME & QUANTITY \\
\hline 1. & Chukku & Zingiber officinale & 1part \\
\hline 2. & Thippili & Piper longum & 1part \\
\hline 3. & Ilavangam & Syzygium aromaticum & 1part \\
\hline 4. & Cirukancori Ver & Tragia involucrate & 1part \\
\hline 5. & Akkirakaram Ver & Anacyclus pyrethrum & 1part \\
\hline 6. & Mulli Ver & Hygrophilla auriculata & 1part \\
\hline 7. & Kadukkaithol & Terminalia chebula & 1part \\
\hline 8. & Adathodai Elai & Adathoda vasica & 1part \\
\hline 9. & Karpooravalli Elai & Coleus amboinicus & 1part \\
\hline 10. & Kostam & Saussurea lappa & 1part \\
\hline 11. & Seenthil Thandu & Tinospora cordifolia & 1part \\
\hline 12. & Siruthekku & Clerodendron serratum & 1part \\
\hline 13. & Nilavembu Samoolam & Andrographis paniculata & 1part \\
\hline 14. & Vattathiruppi Ver & Sida acuta & 1part \\
\hline 15. & Korai Kizhangu & Cyperus rotundus & 1part \\
\hline
\end{tabular}

TABLE: 2 Nilavembu Kudineer Chooranam.

\begin{tabular}{|c|c|c|c|}
\hline S.NO & TAMIL NAME & SCIENTIFIC NAME & QUANTITY \\
\hline 1. & Nilavembu & Andrographis paniculata & 1part \\
\hline 2. & Vettiver & Chrysopogon zizanioides & 1part \\
\hline 3. & Vilamicha ver & Plectranthus vettiveroides & 1part \\
\hline 4. & chandanam & Santalum album & 1part \\
\hline 5. & Korai kizhangu & Cyprus rotundus & 1part \\
\hline 6. & Chukku & Zingiber officinale & 1part \\
\hline 7. & Pei putal & Trichosanthes cucumerina & 1part \\
\hline 8. & Milagu & Pepper nigrum & 1part \\
\hline 9. & Parppatakam & Mollugo cerviana & 1part \\
\hline
\end{tabular}

TABLE: 3 JACOM Chooranam.

\begin{tabular}{|c|c|c|c|}
\hline S.NO & TAMIL NAME & SCIENTIFIC NAME & QUANTITY \\
\hline 1. & Nilavembu & Andrographis paniculata & 1part \\
\hline 2. & Adathodai Elai & Adathoda vasica & 1part \\
\hline 3. & Thulasi & Ocimum tenuiflorum & 1part \\
\hline 4. & Malaivembu & Melia azedarach & 1part \\
\hline 5. & Papali ilai & Carica papaya & 1part \\
\hline
\end{tabular}




\section{EXTRACTION}

The powdered sample of the three polyherbal Siddha formulations was extracted with 500 $\mathrm{mL}$ of aqueous using the Soxhlet apparatus. After completing the extraction process, the extract will be filtered, and the solvent will be removed by distillation under reduced pressure. It will be subjected to neuraminidase inhibition assay.

\section{NEURAMINIDASE INHIBITION ASSAY:}

The NA-FluorTM Influenza Neuraminidase Assay Kit (Life Technologies, No: 4457091) was employed to test the efficacy of aqueous extracts on the viral neuraminidase inactivated H1N1 as per the manufacturer's instructions. The inactivated virus stock was titrated by performing an NA activity assay, and the optimum virus dilution for the neuraminidase inhibition assay was selected. Serial dilutions of aqueous extract of Kabasura Kudineer, Nilavembu Kudineer Chooranam and JACOM $(1280 \mu \mathrm{g} / \mathrm{ml}$ to $2.5 \mu \mathrm{g} / \mathrm{ml})$ were tested for NA inhibitory activity. Oseltamivir was included as a positive control in the assay. Fluorescence was measured using a fluorescence polarization microplate reader (excitation $355 \mathrm{~nm}$, emission $460 \mathrm{~nm} .[4][5]$ Neuraminidase inhibition percentage was determined using doseresponse data using sigmoidal curve fitting with the formula:

\%Activity= Sample intensity /positive control intensity; \% Inhibition = 100-\% Activity

\section{GC-MS analysis}

GC-MS technique was used to analyze the presence of active compounds in the Siddha formulations. Elucidation on the mass spectrum of GC-MS will be prepared using the database NIST08 and WILEY8.[6]

\section{RESULTS \& DISCUSSION}

The minimal inhibitory concentration of Kabasura Kudineer extract was found to be $2.5 \mu \mathrm{g} / \mathrm{ml}$, exhibited $80.35 \%$ of NA inhibition. The maximum inhibition was observed at 1280 $\mu \mathrm{g} / \mathrm{ml}$ and revealed $87.35 \%$ of NA inhibition. (Fig.1). The minimal inhibitory concentration of Nilavembu Kudineer extract was found to be $2.5 \mu \mathrm{g} / \mathrm{mL}$ and presented $91.78 \%$ of NA inhibition. The maximum inhibition was observed at $1280 \mu \mathrm{g} / \mathrm{ml}$ and revealed $90.32 \%$ of NA inhibition. (Fig.2). The minimal inhibition percentage of JACOM at least concentration 2.5 $\mathrm{\mu g} /$ $\mathrm{ml}$ exhibited $87.97 \%$ of inhibition. The maximum was observed at $1280 \mu \mathrm{g} / \mathrm{ml}$ and revealed 88.49\% inhibition (Fig.3). Thus, we conclude that all the two formulations can inhibit 
neuraminidase, among which Nilavembu exhibited maximum activity with the highest inhibition of $91.78 \%$.

The newer class of neuraminidase inhibitors, oseltamivir and zanamivir, has an inhibition range of about $70-80 \%$. Earlier influenza had been treated with adamantanes such as amantadine and rimantadine, which was slightly toxic to the patients. The minimal cytotoxicity was observed in the plant extracts due to cytoprotective components [5]. In our study, this is an indication that these extracts might serve as potential in developing safe and less toxic neuraminidase inhibitor.

The phytochemical compounds resolved in the GC MS analysis revealed active principles in the Kabasura Kudineer, Nilavembu Kudineer and JACOM extracts. (Fig.4, Fig.6 \& Fig.8). GC-MS analysis of Kabasura Kudineer extract showed 6 compounds with 1 major constitutes namely 1,1,1,3,5,7,9,11,11,11 - decamethyl-5-(trimethyl siloxy) hexasiloxane (MW-490). (Fig.5) whereas the Nilavembu Kudineer extract showed 11 compounds with I major constitute as Cyclotetrasiloxane, octamethyl. (Fig.7) and the JACOM extract showed ten compounds with I major constitute as 13208(5-methyl-2-phenyl-1,3-dioxan-4-yl) methanol (Fig.9)

The retention time, molecular mass and chemical structures are represented. (Table.4,5 \& 6) Overall, our study reports provide substantive care for the consumption of three polyherbal Siddha formulations as hopeful sources of novel anti-influenza drug candidates. Future studies are recommended to clinically prove the efficiency of the Nilavembu Kudineer, Kabasura Kudineer Chooranam and JACOM as neuraminidase inhibitors.

\section{Funding information:}

The research is funded by the Central Council for Research in Siddha, Ministry of AYUSH, Government of India.

\section{Competing interest declaration:}

The authors declare that they have no competing interests.

\section{Ethical approval statement:}

NA 


\section{References:}

[1]. Confalonieri U, Menne B, Akhtar R, Ebi KL, Hauengue M, Kovats RS, Revich B, Woodward A. Human health. Climate change 2007: impacts, adaptation and vulnerability: contribution of Working Group II to the fourth assessment report of the Intergovernmental Panel on Climate Change. 2007.

[2] Selvavinayagam TS. Learning from Chennai floods to mitigate epidemic. International Journal of Health System and Disaster Management. 2016 Oct 1;4(4):114.

[3] Bown D. The Royal Horticultural Society encyclopedia of herbs \& their uses. Dorling Kindersley Limited; 1995.

[4]. Li A, Wang W, Xu W, Gong J. A microplate-based screening assay for neuraminidase inhibitors. Drug discoveries \& therapeutics. 2009 Dec 1;3(6).

[5]. Rajasekaran D, Palombo EA, Yeo TC, Ley DL, Tu CL, Malherbe F, Grollo L. Identification of traditional medicinal plant extracts with novel anti-influenza activity. PloS one. 2013 Nov 27;8(11):e79293.

[6] Naine JS, Nasimunislam N, Vaishnavi B, Mohanasrinivasan V, Devi SC. Isolation of soil actinomycetes inhabiting amrithi forest for the potential source of bioactive compounds. Asian J. Pharm. Clin. Res. 2012;5(3):189-92.

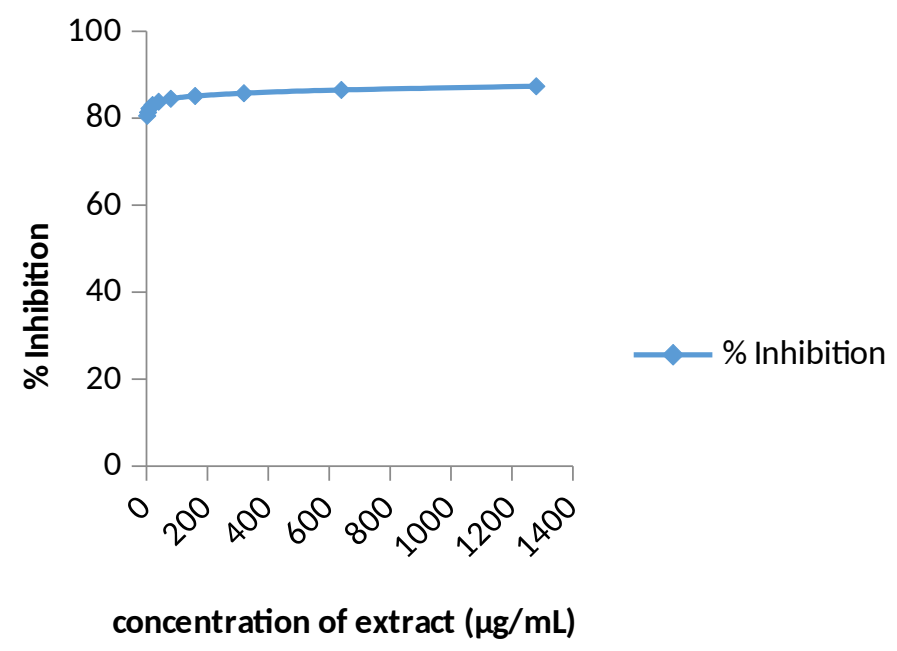

Fig 1: Neuraminidase inhibition by Kabasura Kudineer Chooranam 


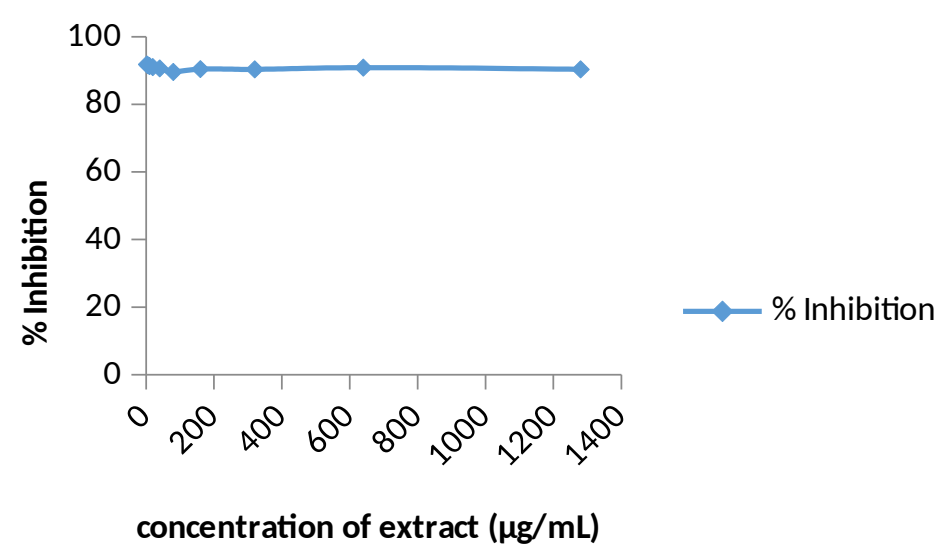

Fig 2: Neuraminidase inhibition by Nilavembu Kudineer Chooranam

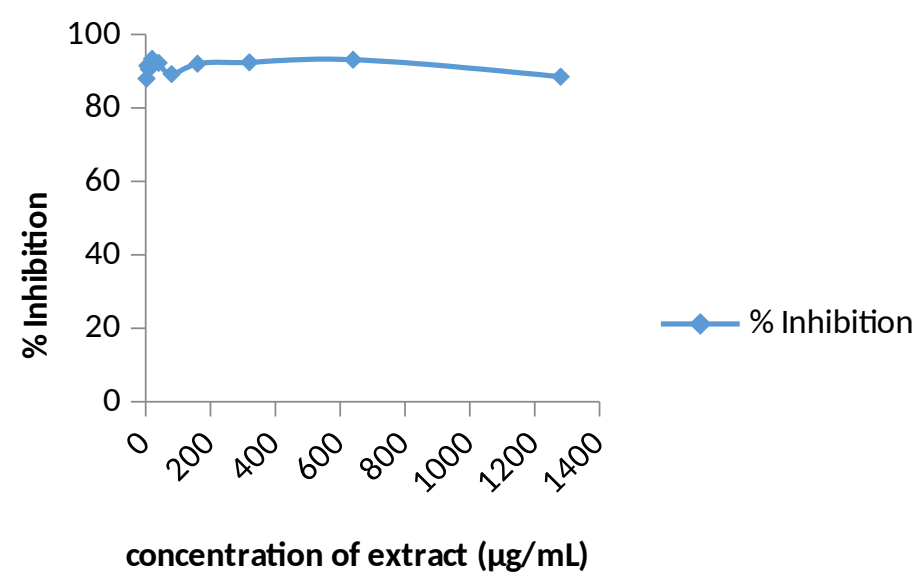

Fig 3: Neuraminidase inhibition by JACOM Chooranam

Table 4: GC-MS analysis of Kabasura Kudineer Chooranam extract

\begin{tabular}{llccc}
\hline S.No. & Compound name & $\begin{array}{c}\text { Retention } \\
\text { time }\end{array}$ & $\begin{array}{c}\text { Molecular } \\
\text { weight }\end{array}$ & $\begin{array}{c}\text { Molecular } \\
\text { formula }\end{array}$ \\
\hline 1 & $\begin{array}{l}1,1,1,3,5,7,9,11,11,11-d e c a m e t h y l-5- \\
\text { (trimethylsiloxy)hexasiloxane }\end{array}$ & 401.1 & 490 & $\mathrm{C}_{13} \mathrm{H}_{42} \mathrm{O}_{6} \mathrm{Si}_{7}$ \\
2 & $\begin{array}{l}\text { Heptasiloxane,1,1,3,3,5,5,7,7,9,9,11,11,13,13- } \\
\text { tetradecamethyl- }\end{array}$ & 407 & 504 & $\mathrm{C}_{14} \mathrm{H}_{44} \mathrm{O}_{6} \mathrm{Si}_{7}$ \\
3 & $\begin{array}{l}\text { Octasiloxane ,1,1,3,3,5,5,7,7,9,9,11,11,13,13, } \\
\text { 15,15-hexadecamethyl- }\end{array}$ & 399 & 578 & $\mathrm{C}_{16} \mathrm{H}_{50} \mathrm{O}_{7} \mathrm{Si}_{8}$ \\
4 & $\begin{array}{l}\text { Cyclohexasiloxane, dodecamethyl- } \\
\text { 1,1,1,5,7,7,7, heptamethyl-3,3- } \\
\text { tris(trimethylsiloxy)tetrasiloxane } \\
\text { Trimethylsilyl-di(timethylsiloxy)-silane }\end{array}$ & 377 & 444 & $\mathrm{C}_{12} \mathrm{H}_{36} \mathrm{O}_{6} \mathrm{Si}_{6}$ \\
& & 349 & 444 & $\mathrm{C}_{13} \mathrm{H}_{40} \mathrm{O}_{5} \mathrm{Si}_{6}$ \\
& & & 280 & $\mathrm{C}_{9} \mathrm{H}_{28} \mathrm{O}_{2} \mathrm{Si}_{4}$ \\
\hline
\end{tabular}




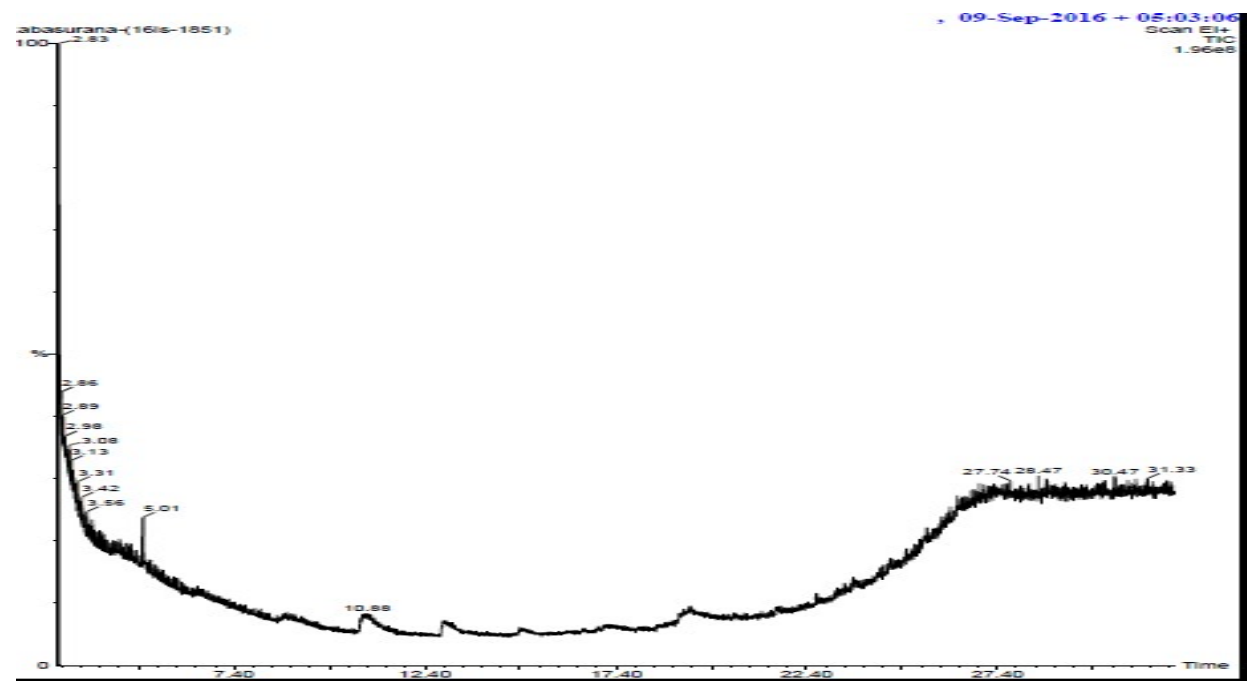

Fig 4: Chromatogram of Kabasura Kudineer Chooranam extract

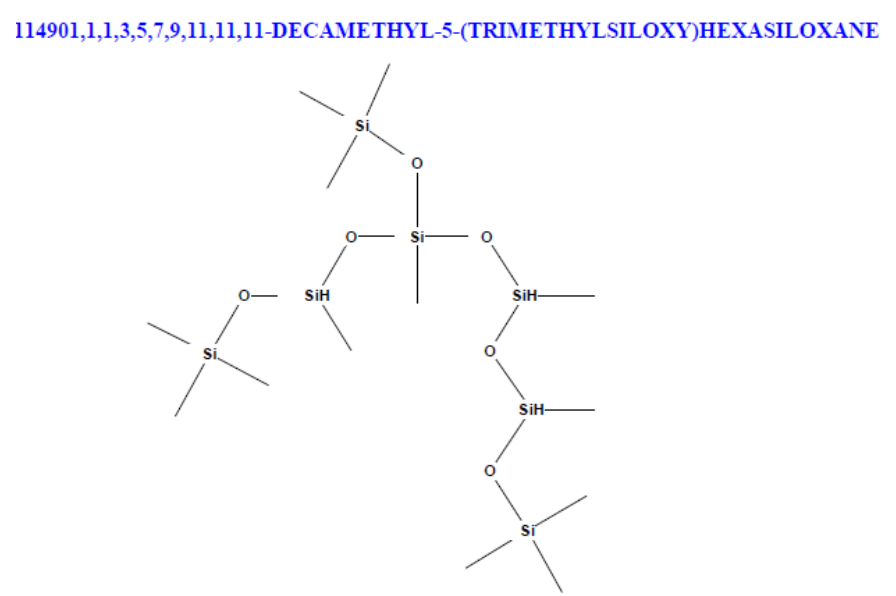

Fig 5: Chemical structure

Table 5: GC-MS analysis of Nilavembu Kudineer Chooranam extract

\begin{tabular}{|c|c|c|c|c|}
\hline S.No & Compound name & $\begin{array}{l}\text { Retentio } \\
\text { n time }\end{array}$ & $\begin{array}{l}\text { Molecula } \\
\text { r weight }\end{array}$ & $\begin{array}{l}\text { Molecular } \\
\text { formula }\end{array}$ \\
\hline 1 & Cyclotetrasiloxane,octamethyl & 735 & 296 & $\mathrm{C}_{8} \mathrm{H}_{24} \mathrm{O}_{4} \mathrm{Si}_{4}$ \\
\hline 2 & $\begin{array}{l}\text { Cyclotetrasiloxane,octamethyl } \\
\text { 2'6'- }\end{array}$ & 704 & 296 & $\mathrm{C}_{8} \mathrm{H}_{24} \mathrm{O}_{4} \mathrm{Si}_{4}$ \\
\hline 3 & $\begin{array}{l}\text { dihydroxyacetophenone,bis(trimethylsilyl)ethe } \\
\mathrm{r}\end{array}$ & 499 & 296 & $\mathrm{C}_{8} \mathrm{H}_{24} \mathrm{O}_{4} \mathrm{Si}_{4}$ \\
\hline 4 & $\begin{array}{l}\text { 2,5- } \\
\text { dihydroxyacetophenone,bis(trimethylsilyl)ethe } \\
\mathrm{r}\end{array}$ & 500 & 296 & $\mathrm{C}_{14} \mathrm{H}_{24} \mathrm{O}_{3} \mathrm{Si}_{2}$ \\
\hline 5 & $\begin{array}{l}\text { 3-ethoxy-1,1,1,5,5,5-hexamethyl-3- } \\
\text { (trimethylsiloxy)teisiloxane }\end{array}$ & 532 & 340 & $\mathrm{C}_{11} \mathrm{H}_{32} \mathrm{O}_{4} \mathrm{Si}_{4}$ \\
\hline
\end{tabular}




\begin{tabular}{|c|c|c|c|c|}
\hline 6 & $\begin{array}{l}\text { Benzoic acid ,4-methyl-2- } \\
\text { trimethylsilyoxy-,trimethylsilyl ester }\end{array}$ & 418 & 296 & $\mathrm{C}_{14} \mathrm{H}_{24} \mathrm{O}_{3} \mathrm{Si}_{2}$ \\
\hline 7 & 5-(p-aminophenyl)-4-(p-tolyl)-2-thiazolamine & 352 & 281 & $\mathrm{C}_{16} \mathrm{H}_{15} \mathrm{~N}_{36}$ \\
\hline 8 & $\begin{array}{l}\text { Benzoic acid ,3-methyl-2- } \\
\text { trimethylsilyoxy-,trimethylsilyl ester }\end{array}$ & 380 & 296 & $\mathrm{C}_{14} \mathrm{H}_{24} \mathrm{O}_{3} \mathrm{Si}_{2}$ \\
\hline 9 & Pentasiloxane ,dodecamethyl- & 412 & 384 & $\mathrm{C}_{12} \mathrm{H}_{36} \mathrm{O}_{4} \mathrm{Si}_{5}$ \\
\hline 10 & $\begin{array}{l}\text { 3-isopropoxy-1,1,1,5,5,5-hexamethyl-3- } \\
\text { (trimethylsiloxy)trisiloxane }\end{array}$ & 410 & 354 & $\mathrm{C}_{12} \mathrm{H}_{36} \mathrm{O}_{4} \mathrm{Si}_{4}$ \\
\hline 11 & $\begin{array}{l}\text { 1-phenazinecarboxilic acid ,6-(1- } \\
\text { methoxyethyl)-methyl ester }\end{array}$ & 319 & 296 & $\mathrm{C}_{17} \mathrm{H}_{16} \mathrm{O}_{3} \mathrm{~N}_{2}$ \\
\hline
\end{tabular}

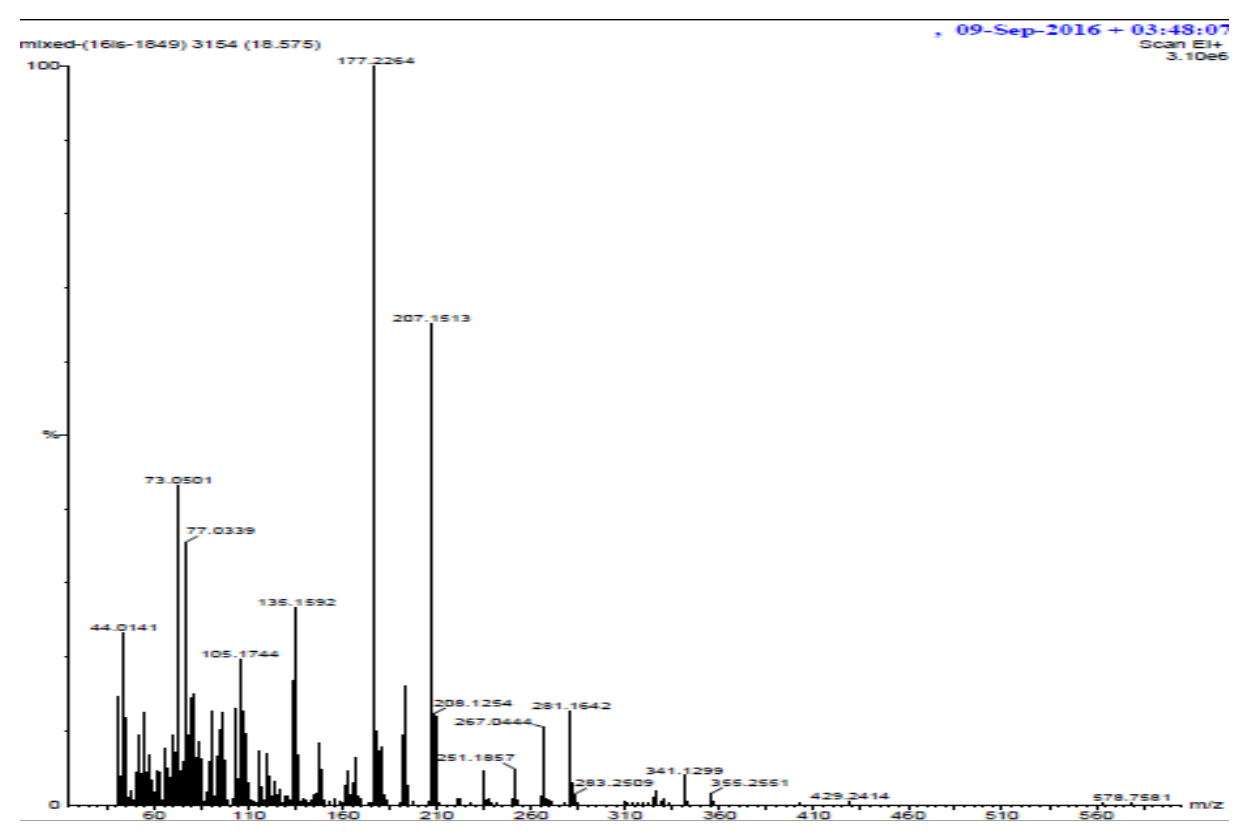

Fig 6: Chromatogram of Nilavembu Kudineer Chooranam extract

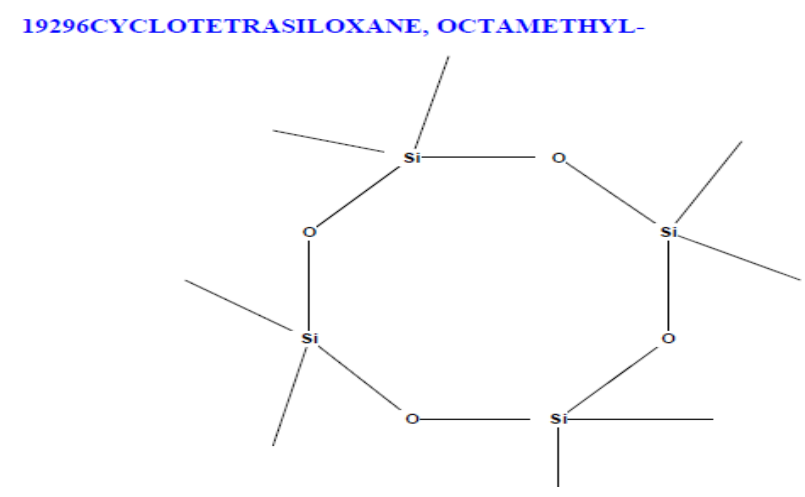

Fig 7: Chemical structure

Table 6: GC-MS analysis of JACOM formulation 


\begin{tabular}{|c|c|c|c|c|}
\hline & & time & weight & formula \\
\hline 1 & $\begin{array}{l}\text { 5-methyl-2-phenyl-1,3-dioxan-4- } \\
\text { yl)methanol }\end{array}$ & 374 & 208 & $\mathrm{C}_{12} \mathrm{H}_{16} \mathrm{O}_{3}$ \\
\hline 2 & $\begin{array}{l}\text { 1,2-dimethoxy-4-(1-methoxy-1- } \\
\text { propenyl)benzene }\end{array}$ & 401 & 613 & $\mathrm{C}_{12} \mathrm{H}_{16} \mathrm{O}_{3}$ \\
\hline 3 & $\begin{array}{l}\text { 1,4-cyclohexandiene-1,2-dicarboxylic } \\
\text { acid ,4,5-dimethyl-dimethyl est }\end{array}$ & 330 & 611 & $\mathrm{C}_{12} \mathrm{H}_{16} \mathrm{O}_{3}$ \\
\hline 4 & $\begin{array}{l}\text { Benzeneacetic acid ,alpha-oxo- } \\
\text { trimethylsilyl ester }\end{array}$ & 377 & 222 & $\mathrm{C}_{11} \mathrm{H}_{14} \mathrm{O}_{3} \mathrm{Si}$ \\
\hline 5 & $\begin{array}{l}\text { 2-thiazolamine,n-[2-(3,4- } \\
\text { dimethoxyphenyl)ethyl]-4-(2-pyrinyl)- }\end{array}$ & 305 & 341 & $\mathrm{C}_{18} \mathrm{H}_{19} \mathrm{O}_{2} \mathrm{~N}_{3} \mathrm{~S}$ \\
\hline 6 & $\begin{array}{l}\text { 1(3H)-isobenzofuranone,6,7-dimethoxy-3- } \\
\text { [2-(2-methoxyphenyl)-2-oxoe }\end{array}$ & 328 & 342 & $\mathrm{C}_{19} \mathrm{H}_{18} \mathrm{O}_{6}$ \\
\hline 7 & Phenol,2,4-bis(1-methylpropyl)- & 300 & 206 & $\mathrm{C}_{14} \mathrm{H}_{22} \mathrm{O}$ \\
\hline 8 & 2,4-benzyldiene-d-glucose & 317 & 268 & $\mathrm{C}_{13} \mathrm{H}_{16} \mathrm{O}_{6}$ \\
\hline 9 & N-methyl-1-adamantaneacetamide & 348 & 207 & $\mathrm{C}_{13} \mathrm{H}_{21} \mathrm{ON}$ \\
\hline 10 & $\begin{array}{l}\text { Androstane-11,17-dione,3- } \\
\text { [(trimethylsilyl)oxy]-17-[o-(phenylmethyl) }\end{array}$ & 323 & 481 & $\mathrm{C}_{29} \mathrm{H}_{43} \mathrm{O}_{3} \mathrm{NSi}$ \\
\hline
\end{tabular}

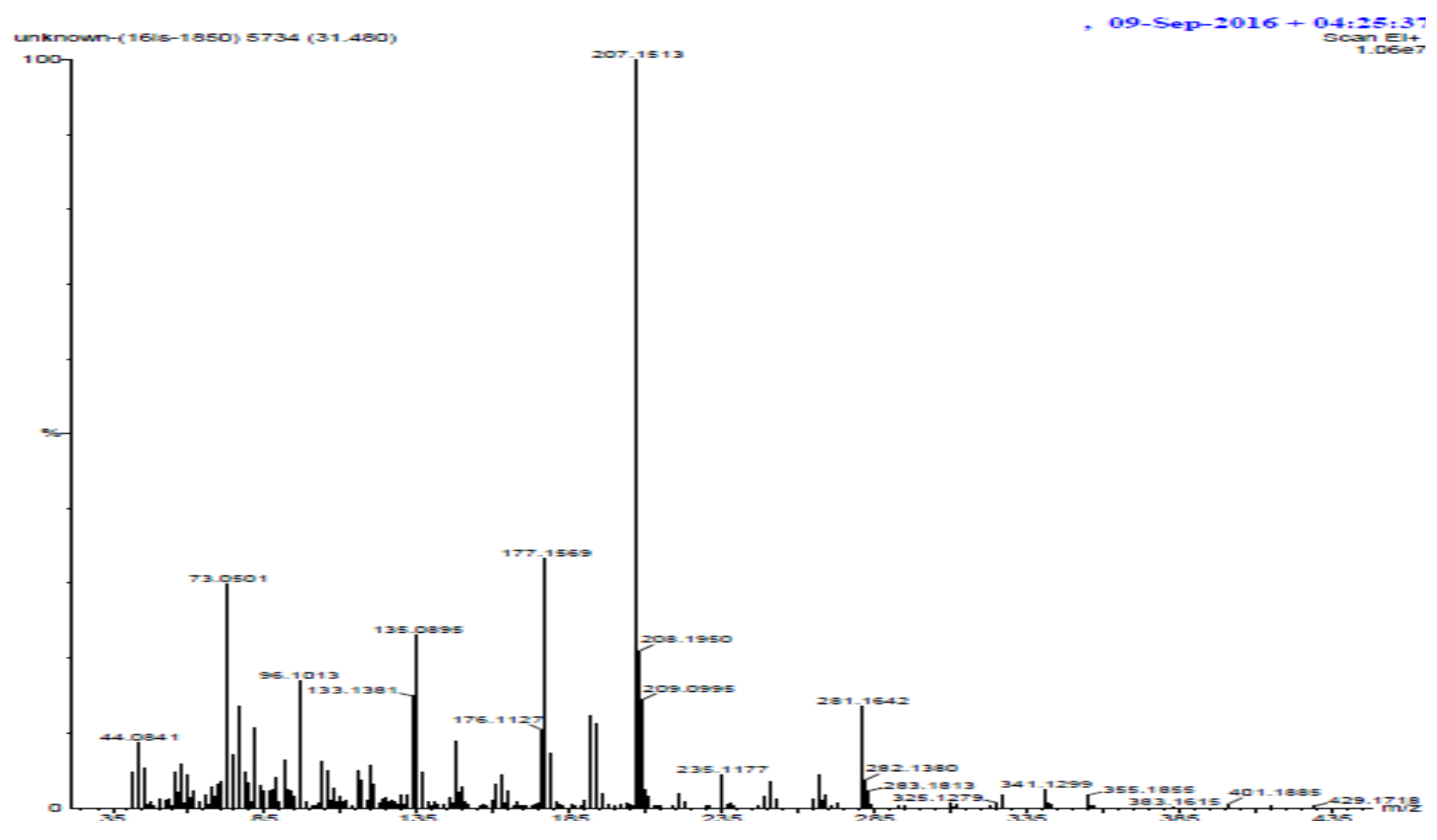

Fig 8: Chromatogram of JACOM 
13208(5-METHYL-2-PHENYL-1,3-DIOXAN-4-YL)METHANOL

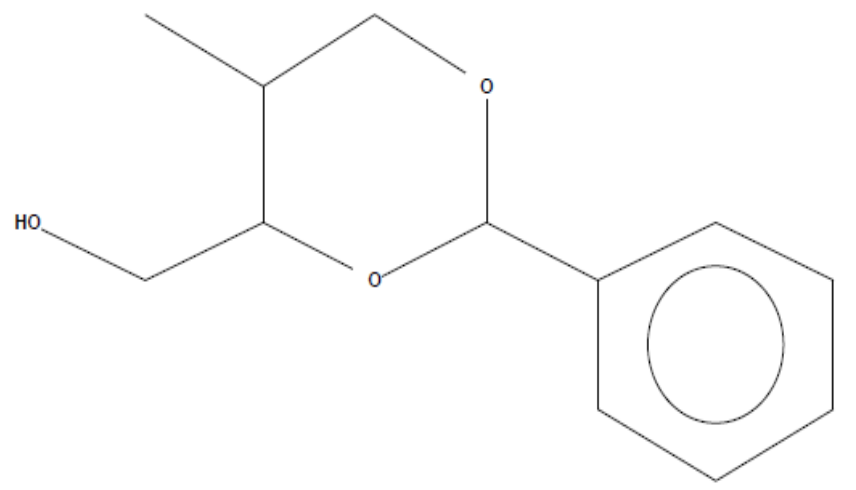

Fig 9: Chemical structure 